\title{
An improved method for the conversion of oxiranes to thiiranes and the discrimination of their base peaks in EI-MS
}

\author{
Hsing-Ming Chen, ${ }^{a}$ Po-Yuan Chen, ${ }^{b}$ Chih-Feng Wang, ${ }^{c}$ Jui-Chi Tsai, ${ }^{b}$ and Eng-Chi Wang ${ }^{b} *$ \\ ${ }^{a}$ Department of Nutrition and Health Science, Fooyin University, Kaohsiung 831, Taiwan \\ ${ }^{b}$ Department of Medicinal and Applied Chemistry, Kaohsiung Medical University, Kaohsiung \\ 807, Taiwan \\ ${ }^{c}$ Department of Materials Science \& Engineering, I-Shou University, Kaohsiung 82445, Taiwan \\ E-mail: enchwa@kmu.edu.tw
}

\begin{abstract}
An efficient method for the conversion of oxiranes to thiiranes by treatment with excess ammonium thiocyanate in aqueous media under reflux or by microwave irradiation is reported.
\end{abstract}

Keywords: Epoxides, ammonium thiocyanate, thiiranes, base peak, EI-MS

\section{Introduction}

Thiiranes are valuable synthetic building blocks with industrial potential for the preparation of polymers, ${ }^{1}$ liquid crystals, ${ }^{2}$ adhesives, ${ }^{3}$ as many others. ${ }^{4}$ In addition, thiirane is an essential heterocyclic core of various compounds with biological activity including selective gelatinase inhibitors, ${ }^{5}$ selective $\mathrm{A}_{1}$ adenosine receptor agonists, ${ }^{6}$ potential topoisomerase I inhibitors, ${ }^{7}$ estrogen synthetase inhibitor ${ }^{8}$. As a result chemists have made efforts to create an efficient and environmentally benign method for the synthesis of thiiranes. Strategies for the preparation of thiiranes include (1) reaction of alkenes with various reagents including dichlorodisulfides, ${ }^{9}$ and iodothiocyanate, $^{10}$ (2) reaction of ketones with a sulfuryl lithium, ${ }^{11}$ (3) reduction of 1 (benzthiazol-2-yl-sulfanyl)-2-alkanones with sodium hydride, ${ }^{12}$ (4) reaction of epoxides with ammonium thiocyanate, ${ }^{13}$ or potassium thiocyanate ${ }^{14}$ (5) conversion of epoxides with thiourea, ${ }^{15}$ as well as other methods. ${ }^{16}$ Among the methods described above, preparation of thiiranes from epoxides, by reaction ammonium thiocyanate with various catalysts and solvents, has been intensively studied and summarized in Table 1.

Although numerous reaction conditions have been investigated, disadvantages such reaction time, use of environmentally unfriendly solvents and toxic heavy metal catalysts remain to be addressed. Herein we disclose a method to convert epoxides to thiiranes in aqueous media either by heating under reflux or by microwave irradiation with varying wave lengths. The method 
requires no metallic catalyst or organic solvent and the base peaks of resulting thiiranes in EI-MS are also discussed.

Table 1. Reported methods for the conversion of epoxides to thiiranes by ammonium thiocyanate with various conditions

\begin{tabular}{|c|c|c|c|c|c|c|}
\hline Reagent & $\begin{array}{c}\text { Solvent } \\
\text { (Co-solvent) }\end{array}$ & Catalyst & $\begin{array}{l}\text { Reaction } \\
\text { Temp. }\end{array}$ & $\begin{array}{c}\text { Reaction } \\
\text { Time (min.) }\end{array}$ & $\begin{array}{c}\text { Yields } \\
(\%)\end{array}$ & Ref. \\
\hline $\mathrm{NH}_{4} \mathrm{SCN}$ & $\mathrm{H}_{2} \mathrm{O}, \mathrm{NaOH}$ & PVA \& PAA & $\begin{array}{l}25 \text { or } \\
45^{\circ} \mathrm{C}\end{array}$ & $\begin{array}{l}30-100 \\
\text { min. }\end{array}$ & $86-96$ & $13 a$ \\
\hline $\mathrm{NH}_{4} \mathrm{SCN}$ & $\mathrm{CH}_{3} \mathrm{CN}$ & $\mathrm{RuCl}_{3}$ & $\begin{array}{l}25 \text { or } \\
80^{\circ} \mathrm{C}\end{array}$ & $\begin{array}{l}15-120 \\
\min .\end{array}$ & $93-100$ & $13 b$ \\
\hline $\mathrm{NH}_{4} \mathrm{SCN}$ & tert-BuOH & CAN & $\mathrm{rt}$ & $15-480 \mathrm{~min}$ & $90-100$ & $13 c$ \\
\hline $\mathrm{NH}_{4} \mathrm{SCN}$ & $\mathrm{CH}_{3} \mathrm{CN}$ & Oxalic acid & Reflux & 30 min. & 95 & $13 d$ \\
\hline $\mathrm{NH}_{4} \mathrm{SCN}$ & tert-BuOH & $\mathrm{LiBF}_{4}$ & Reflux & $20 \mathrm{~min}$. & 95 & $13 \mathrm{e}$ \\
\hline $\mathrm{NH}_{4} \mathrm{SCN}$ & $\mathrm{CH}_{3} \mathrm{CN}$ & $\mathrm{Bi}(\mathrm{OTf})_{3}$ & $20^{\circ} \mathrm{C}$ & $15 \min$. & 98 & $13 f$ \\
\hline $\mathrm{NH}_{4} \mathrm{SCN}$ & $\mathrm{CH}_{3} \mathrm{CN}$ & $\begin{array}{c}\left(\mathrm{NH}_{4}\right)_{8}\left[\mathrm{CeWO}_{36}\right] \\
20 \mathrm{H}_{2} \mathrm{O}\end{array}$ & Reflux & $0.5-3.5 \mathrm{hr}$ & $88-98$ & $13 \mathrm{~g}$ \\
\hline $\mathrm{NH}_{4} \mathrm{SCN}$ & $\mathrm{CH}_{3} \mathrm{CN}$ & $\begin{array}{c}\text { Iron (III) } \\
\text { trifluoroacetate }\end{array}$ & $\mathrm{rt}$ & $30-60 \mathrm{~min}$ & $91-98$ & $13 \mathrm{~h}$ \\
\hline $\mathrm{NH}_{4} \mathrm{SCN}$ & $\mathrm{CH}_{3} \mathrm{CN}$ & $\mathrm{SbCl}_{3}$ & Reflux & $25-40 \mathrm{~min}$ & $95-98$ & $13 \mathrm{i}$ \\
\hline $\mathrm{NH}_{4} \mathrm{SCN}$ & $\mathrm{CH}_{3} \mathrm{CN}$ & $\mathrm{P} 4 \mathrm{VP}-\mathrm{Ce}(\mathrm{OTf})_{4}$ & $\mathrm{rt}$ & 15-40 min. & $85-92$ & $13 \mathrm{j}$ \\
\hline $\mathrm{NH}_{4} \mathrm{SCN}$ & $\mathrm{CH}_{3} \mathrm{CN}$ & $\mathrm{BiCl}_{3}$ & Reflux & 10-35 min. & $96-99$ & $13 \mathrm{k}$ \\
\hline $\mathrm{NH}_{4} \mathrm{SCN}$ & $\mathrm{CH}_{3} \mathrm{CN}$ & $\mathrm{TiO}(\mathrm{TFA})_{3}$ & Reflux & $15-40 \mathrm{~min}$. & $90-97$ & 131 \\
\hline
\end{tabular}

\section{Results and Discussion}

In our studies, (Table 2) we found that thiiranes can be efficiently obtained from the reaction of epoxide and excess ammonium thiocyanate (2 equv.) in aqueous media by microwave irradiation. Heating under reflux results in a longer reaction time and lower yields than for microwave irradiation. For example, in entry 3, under reflux conditions, it took 3 hours to completely consume the starting styrene oxide (1c), and only trace of styrene thiirane (2c) was formed. In contrast, styrene thiirane (2c) was obtained in $92 \%$ yield by microwave irradiation using a $160 \mathrm{~W}$ source. Furthermore, in entry 4, it took 4 hours to give 2-(naphthalene-1oxymethyl)thiirane (1d) in 53\% yield under reflux conditions, but only 45 min to produce 2(naphthalene-1-oxymethyl)thiirane (2d) in $90 \%$ yield by microwave irradiation using a $300 \mathrm{~W}$ source. 
Table 2. The comparison of conversion of epoxides to thiiranes with ammonium thiocyanate in aqueous media either by reflux or by microwave irradiation

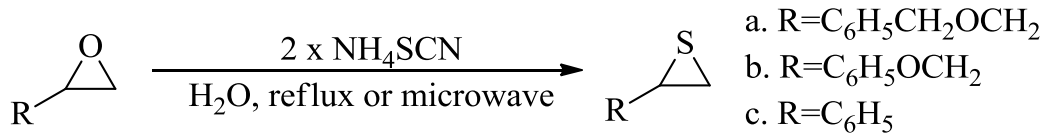

$$
\begin{aligned}
& 1 \\
& \text { d. } \mathrm{R}=1 \text {-naphthalenoxymethyl } \\
& \text { e. } \mathrm{R}=4 \text {-methoxyphenoxymethyl } \\
& \text { f. } \mathrm{R}=3 \text {,4-dimethoxyphenoxymethyl } \\
& \text { g. } \mathrm{R}=3 \text {-isopropoxy-4-methoxyphenoxymethyl } \\
& \text { h. } R=3,4 \text {-methylenedioxyphenylmethyl }
\end{aligned}
$$

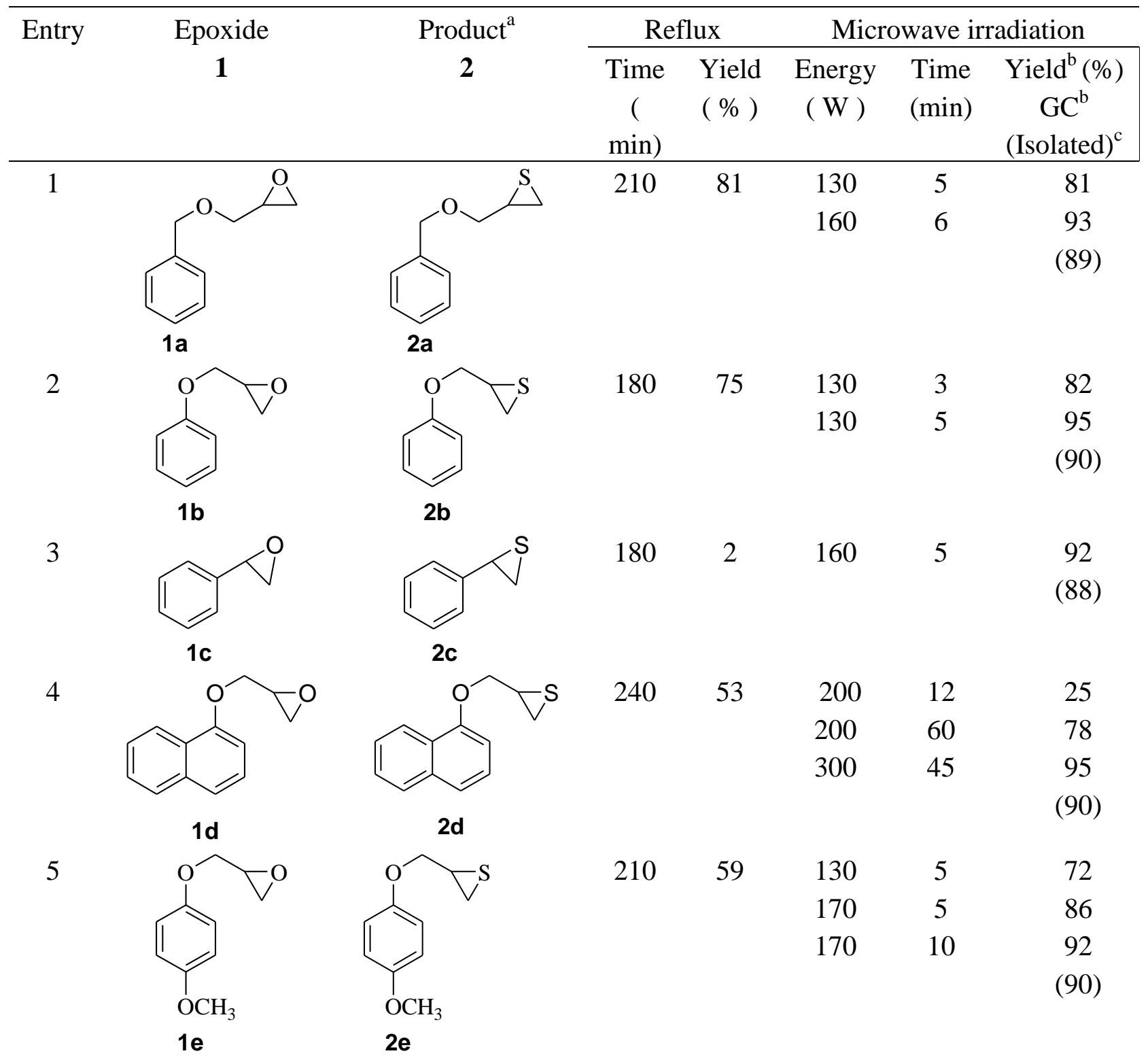


Table 2 (continued)

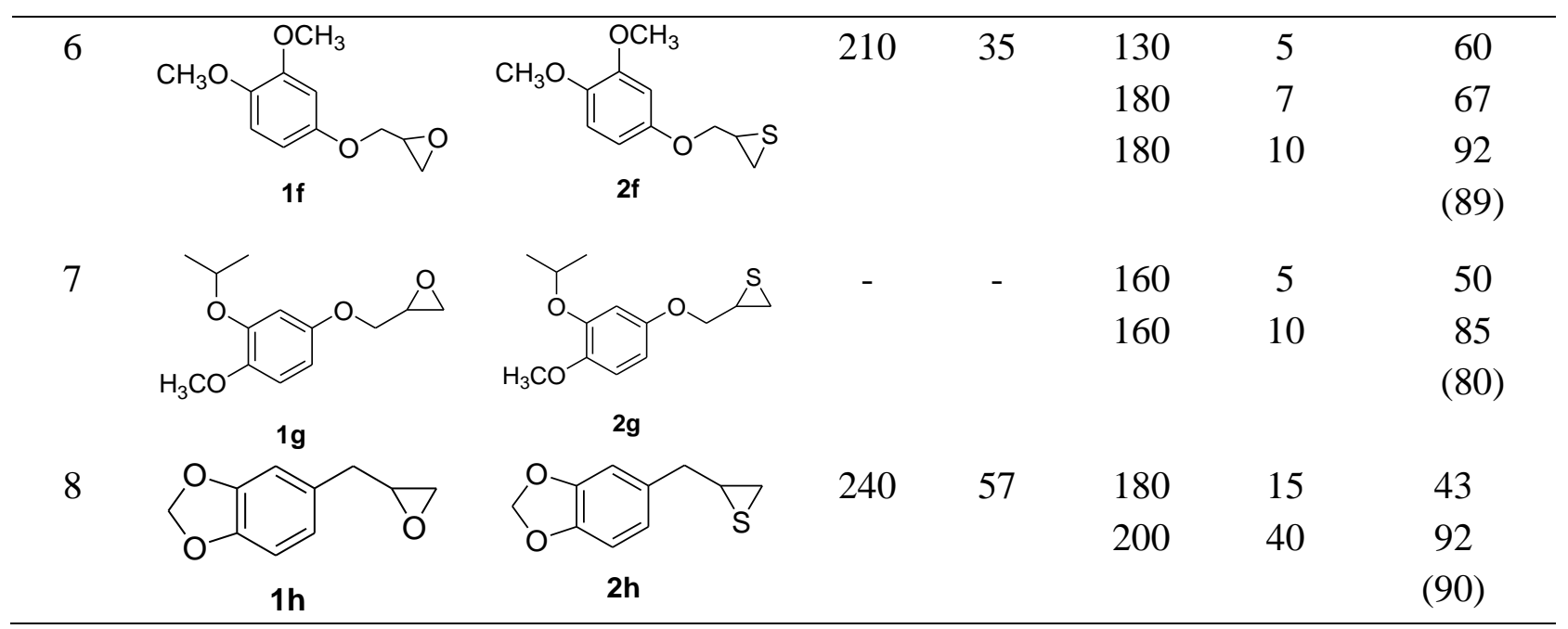

${ }^{a}$ All thiiranes prepared have satisfactory spectral data; thiiranes $\mathbf{2 a}, \mathbf{2 b}, \mathbf{2 c}, \mathbf{2 d}$, and $\mathbf{2 e}$ are known compounds, but $\mathbf{2 f}, \mathbf{2 g}$, and $\mathbf{2 h}$ are new. ${ }^{\mathrm{b}}$ Determined by gas chromatography. ${ }^{\mathrm{c}}$ Isolated from silica gel column chromatography. ${ }^{-}$The reflux reaction condition was skipped for no enough starting material.

The structures of thiiranes (2a-h) were confirmed by ${ }^{1} \mathrm{H}-\mathrm{NMR}$ and ${ }^{13} \mathrm{C}-\mathrm{NMR}$ (Table 3). The EI-MS, HRMS of $\mathbf{2 a - h}$ and elemental analysis of $\mathbf{2 d - h}$ are reported in Table $\mathbf{4}$.

Table 3. The Selected Signals of ${ }^{1} \mathrm{H}-\mathrm{NMR}$ and ${ }^{13} \mathrm{C}-\mathrm{NMR}$ spectra of thiiranes (2a-h)

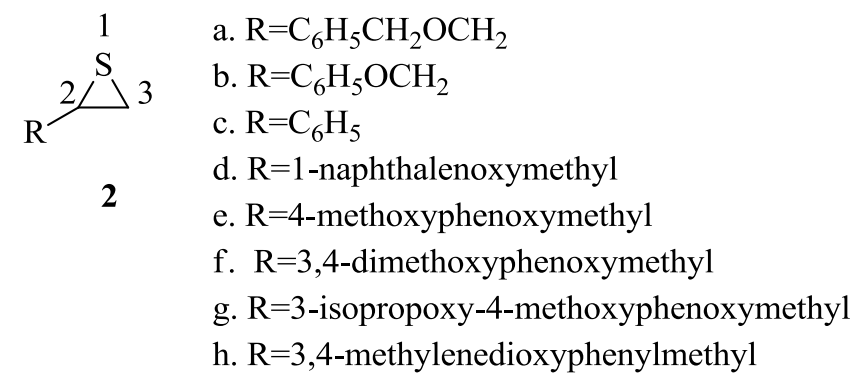

\begin{tabular}{ccl}
\hline Thiiranes & $\begin{array}{c}\text { Selected signals of }{ }^{1} \mathrm{H}-\mathrm{NMR} \\
\left(\mathrm{CDCl}_{3}, 200 \mathrm{MHz}\right)\end{array}$ & ${ }^{13} \mathrm{C}-\mathrm{NMR}\left(\mathrm{CDCl}_{3}, 50 \mathrm{MHz}\right)$ \\
\hline $\mathbf{2 a}$ & $2.18\left(\mathrm{dd}, J 5.2,1.2 \mathrm{~Hz}, 1 \mathrm{H}, \mathrm{H}_{\mathrm{a}}-3\right)$, & $23.7,32.1,73.0,74.5,76.4,127.6,1$ \\
$(\mathrm{R}=$ & & $127.7,128.3,137.8$. \\
$\left.\mathrm{C}_{6} \mathrm{H}_{5} \mathrm{CH}_{2} \mathrm{OCH}_{2}\right)$ & $2.48(\mathrm{dd}, J 6.0,1.2 \mathrm{~Hz}, 1 \mathrm{H}, \mathrm{Hb}-3)$, \\
& $3.08(\mathrm{~m}, 1 \mathrm{H}, \mathrm{H}-2)$, \\
& $3.47\left(\mathrm{dd}, J 10.8,6.0 \mathrm{~Hz}, 1 \mathrm{H}, \mathrm{H}_{\mathrm{a}}-4\right)$, \\
& $3.66\left(\mathrm{dd}, J 10.8,5.2 \mathrm{~Hz}, 1 \mathrm{H}, \mathrm{H}_{\mathrm{b}}-4\right)$.
\end{tabular}


Table 3 (continued)

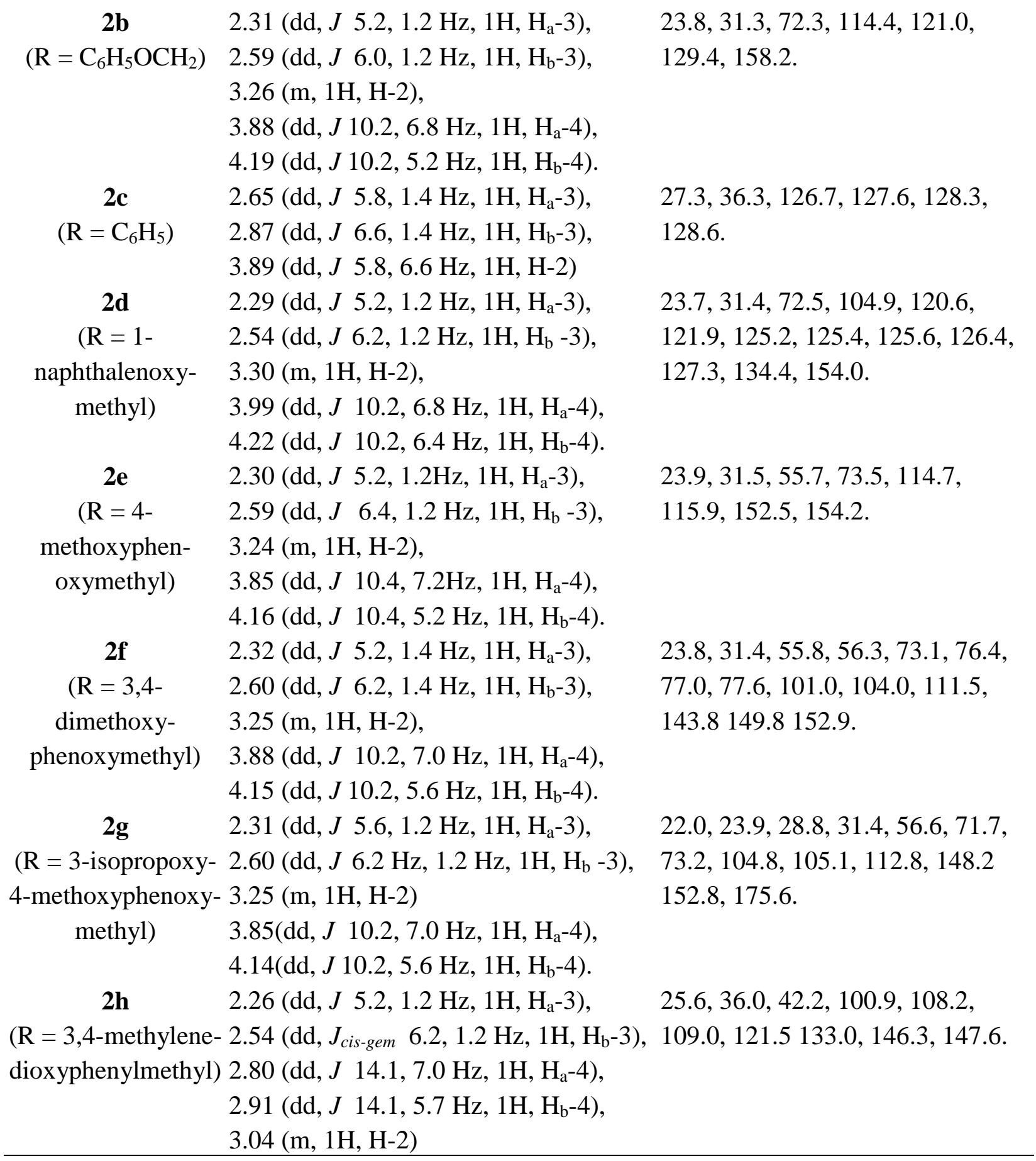


Table 4. The EI-MS, HRMS and EA data of thiiranes (2a-h)

\begin{tabular}{|c|c|c|c|c|c|}
\hline Thiiranes & EI-MS & HRMS & \multicolumn{3}{|c|}{$\begin{array}{c}\text { Elemental Analysis* } \\
\% \text { Calcd (Found) }\end{array}$} \\
\hline $2 \mathbf{a}$ & 181( $\left(\mathrm{M}^{+1} 68\right)$ & Calcd Found & $\mathrm{C}$, & $\mathrm{H}$, & $\mathrm{S}$ \\
\hline $\begin{array}{c}(\mathrm{R}= \\
\left.\mathrm{C}_{6} \mathrm{H}_{5} \mathrm{CH}_{2} \mathrm{OCH}_{2}\right)\end{array}$ & $\begin{array}{l}\text { 163(30), 107(39), } \\
\text { 92(42), 91(100), } \\
\text { 79(17). }\end{array}$ & $\begin{array}{l}180.0603 \\
180.0603\end{array}$ & -- & -- & -- \\
\hline $\begin{array}{c}\mathbf{2 b} \\
\left(\mathrm{R}=\mathrm{C}_{6} \mathrm{H}_{5} \mathrm{OCH}_{2}\right)\end{array}$ & $\begin{array}{l}\left.\text { 166( } \mathrm{M}^{+}, 37\right) \\
\text { 165(30), 133(17), } \\
\text { 74(19), 73(100). }\end{array}$ & $\begin{array}{c}\text { Calcd Found } \\
166.0447 \\
166.0449\end{array}$ & $\begin{array}{l}\mathrm{C}, \\
--\end{array}$ & $\begin{array}{l}\mathrm{H}, \\
--\end{array}$ & $\begin{array}{l}S \\
--\end{array}$ \\
\hline $\begin{array}{c}\mathbf{2 c} \\
\left(\mathrm{R}=\mathrm{C}_{6} \mathrm{H}_{5}\right)\end{array}$ & $\begin{array}{l}\left.\text { 136( } \mathrm{M}^{+}, 81\right), \\
135(100), \\
104(14), 91(44)\end{array}$ & $\begin{array}{c}\text { Calcd Found } \\
136.0341 \\
136.0342\end{array}$ & $\begin{array}{l}\mathrm{C}, \\
--\end{array}$ & $\begin{array}{l}\mathrm{H}, \\
--\end{array}$ & $\begin{array}{l}S \\
--\end{array}$ \\
\hline $\begin{array}{c}\mathbf{2 d} \\
(\mathrm{R}=1-\text {-naph } \\
\text { thalenoxy- } \\
\text { methyl) }\end{array}$ & $\begin{array}{l}216\left(\mathrm{M}^{+} 34\right), \\
116(22), 115(58), \\
73(100) .\end{array}$ & $\begin{array}{c}\text { Calcd Found } \\
216.0603 \\
216.0605\end{array}$ & $\begin{array}{c}\mathrm{C} \\
72.19 \\
(72.20)\end{array}$ & $\begin{array}{l}\mathrm{H}, \\
5.59 \\
(5.62)\end{array}$ & $\begin{array}{c}\mathrm{S} \\
14.82 \\
(14.80)\end{array}$ \\
\hline $\begin{array}{c}\mathbf{2 e} \\
(\mathrm{R}=4- \\
\text { methoxyphen- } \\
\text { oxymethyl) }\end{array}$ & $\begin{array}{l}\left.\text { 196( } \mathrm{M}^{+}, 26\right), \\
123(33), 95(28), \\
73(100) .\end{array}$ & $\begin{array}{c}\text { Calcd Found } \\
196.0553 \\
196.0555\end{array}$ & $\begin{array}{l}\text { C } \\
61.20 \\
(61.30)\end{array}$ & $\begin{array}{c}\mathrm{H}, \\
6.16 \\
(6.22)\end{array}$ & $\begin{array}{c}S \\
16.34 \\
(16.56)\end{array}$ \\
\hline $\begin{array}{c}\mathbf{2 f} \\
(\mathrm{R}=3,4- \\
\text { dimethoxy- } \\
\text { phenoxymethyl })\end{array}$ & $\begin{array}{l}226\left(\mathrm{M}^{+}, 83\right), \\
154(53), 153(41), \\
\text { 125(55), 74(38), } \\
73(100) .\end{array}$ & $\begin{array}{c}\text { Calcd Found } \\
226.0658 \\
226.0659\end{array}$ & $\begin{array}{c}\text { C, } \\
58.38 \\
(58.39)\end{array}$ & $\begin{array}{l}\mathrm{H}, \\
6.24 \\
(6.26)\end{array}$ & $\begin{array}{c}\mathrm{S} \\
14.17 \\
(14.16)\end{array}$ \\
\hline $\begin{array}{c}\mathbf{2 g} \\
(\mathrm{R}=3- \\
\text { isopropoxy-4- } \\
\text { methoxyphenoxy } \\
\text { methyl) } \\
\mathbf{2 h} \\
(\mathrm{R}=3,4- \\
\text { methylene- } \\
\text { dioxyphenylmeth } \\
\text { yl) }\end{array}$ & $\begin{array}{l}254\left(\mathrm{M}^{+}, 36\right), \\
222(15), 212(27), \\
\text { 182(22), 165 (39), } \\
\text { 140(47), 139(63), } \\
\text { 111(43), 73(100). } \\
\text { 194( }\left(\mathrm{M}^{+}, 100\right), \\
161(36), 135(29), \\
131(24), 103(19), \\
77(12) . .\end{array}$ & $\begin{array}{c}\text { Calcd Found } \\
254.0971 \\
254.0973\end{array}$ & $\begin{array}{l}\text { C } \\
61.39 \\
(61.51)\end{array}$ & $\begin{array}{l}\mathrm{H}, \\
7.13 \\
(7.29)\end{array}$ & $\begin{array}{l}\mathrm{S} \\
12.61 \\
(12.69)\end{array}$ \\
\hline
\end{tabular}

*The elemental analysis of thiiranes $\mathbf{2 d}, \mathbf{2 f}, \mathbf{2 g}$, and $\mathbf{2 h}$ are measured. 
The thiiranes have a characteristic base peak in EI-MS. For example, the fragment of $\mathrm{m} / \mathrm{z}, 73$ in compound $\mathbf{2 b}, \mathbf{2 d}, \mathbf{2 e}, \mathbf{2 f}$, and $\mathbf{2 g}$; but $\mathrm{m} / \mathrm{z} 91$ in compound $\mathbf{2 a}, \mathrm{m} / \mathrm{z} 135$ in compound $\mathbf{2 c}$, and $\mathrm{m} / \mathrm{z} 194\left(\mathbf{M}^{+}\right)$in compound $\mathbf{2 h}$ was found as base peak. The resulting thiiranes $(\mathbf{2} \mathbf{a}-\mathbf{h})$ could be classified into four categories based on their base peaks in EI/MS: i) benzyloxymethylthiirane (2a), ii) aryloxymethylthiirane (2b, 2d, 2e, 2f and $\mathbf{2 g}$ ), iii) phenylthiirane (2c), and iv) $O$ functional arymethylthiirane (2h). The base peak in EI-MS can be used as for structural identification. The mechanism for formation of the base peak in each category of thiiranes (2a-h) is proposed. The formation of base peak $\mathrm{m} / \mathrm{z} 91$ of benzyloxymethylthiirane (2a) involves the loss of a radical from the oxymethylenethiiryl group when initially impacted by an electron, and subsequent cleavage of the $\mathrm{C}-\mathrm{O}$ bond to generate the benzylic cation which spontaneously converts to a stable tropyllium ion $m / z$, 91, equation 1 .

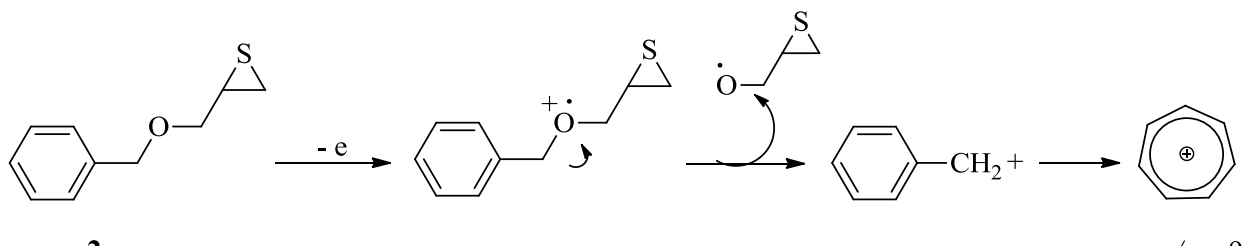

The formation of the base peak $m / z 73$ is common to aryloxymethylthiiranes $\mathbf{2 b}, \mathbf{2 d}, \mathbf{2 e}$, and 2f, equation 2 .

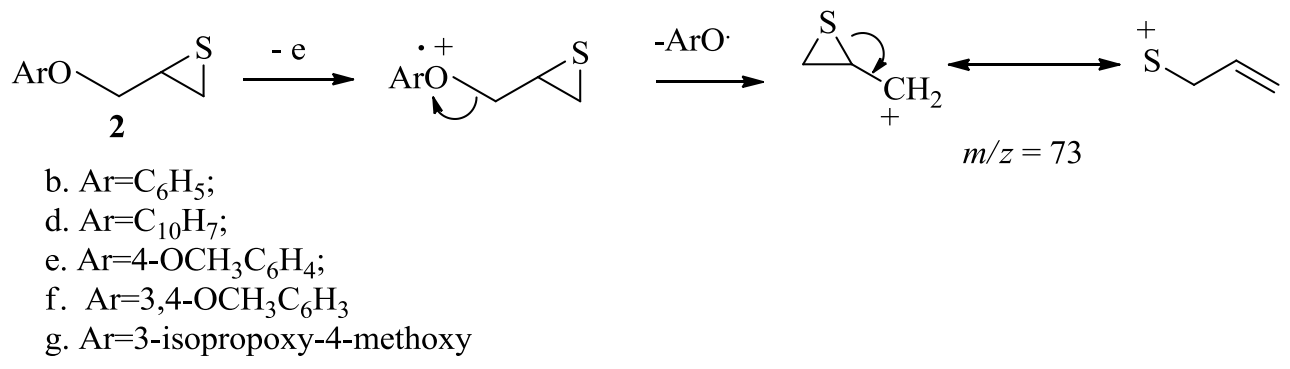

The formation of base peak $\mathrm{m} / \mathrm{z} 135$ of phenylthiiranes (2c) is proposed to result as shown in equation 3 , from molecular ion, $m / z 136$ by the loss of one hydrogen radical to generate the resonance stablisied phenyl thiirene cation, $m / z 135$.

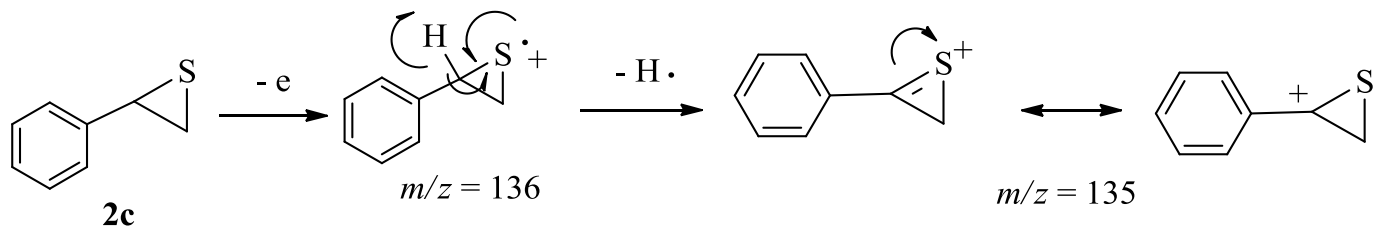

The formation of base peak of $O$-functional arymethylthiirane (2h) is proposed to result as shown in equation 4. 


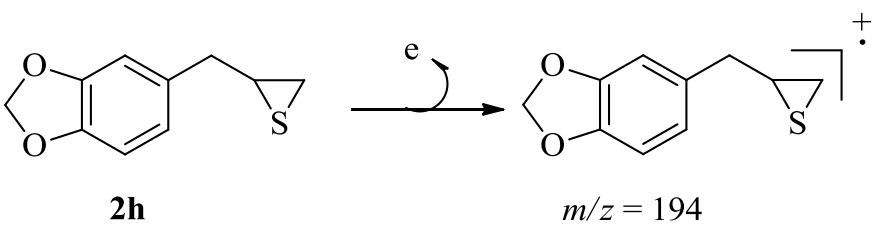

\section{Conclusions}

In general in aqueous ammonium thiocyanate solution, epoxides can be converted into thiiranes, by heating under reflux or in higher yield by microwave irradiation .The formation of base peak in EI-MS of resulting the thiiranes was rational.

\section{Experimental Section}

General. Melting points (Yanaco micro melting-point apparatus) were uncorrected. ${ }^{1} \mathrm{H}-\mathrm{NMR}$ and ${ }^{13}$ C-NMR spectra were obtained on a Varian Gemini-200 or Varian Unity plus 400. Chemical shifts were measured in parts per million with respect to TMS. MS spectra were recorded on a Chem/hp/middle instrument. HRMS spectra were performed on a JEOL JMS SX/SX 102A instrument. Elemental analyses were recorded on a Heraeus CHN-O Rapid analyzer. Silica gel (230-400 mesh) for column chromatography and the precoated silica gel plate (60 F-254) for TLC were purchased from E. Merck Co. UV light (254 nm) was used to detect spots on TLC plates after development. Microwave irritating was carried on CEM, Discover or PROLABO, SYNTHEWAVE 402 microwave apparatus.

Benzyl glycidyl ether (1a) was prepared according to the known procedure, ${ }^{17}$ epoxides (1b, 1d1f) were prepared from the epoxidation of corresponding allyl compounds with $m \mathrm{CPBA}$ in dichloromethane at $0^{\circ} \mathrm{C}$, and epoxides $1 \mathrm{c}$ and $1 \mathrm{~g}$ were purchased from Aldrich.

\section{General procedure for the conversion of oxiranes (1a-g) to thiiranes (2a-h)}

The mixture of epoxide (20 mmol), $\mathrm{NH}_{4} \mathrm{SCN}$ (3.04 g, $40 \mathrm{mmol}$ ), and $\mathrm{H}_{2} \mathrm{O}(200 \mathrm{~mL})$ was stirred and heated to reflux or the mixture of epoxide ( $2 \mathrm{mmol}), \mathrm{NH}_{4} \mathrm{SCN}(0.3 \mathrm{~g}, 4.0 \mathrm{mmol})$, and $\mathrm{H}_{2} \mathrm{O}$ (30 $\mathrm{mL}$ ) was heated and irradiated on the microwave reactor with various reaction time, and energy (130 W to $300 \mathrm{~W}$, see Table 2). When the starting material, epoxide, was completely consumed, the resulting mixture was extracted with EtOAc $(20 \mathrm{~mL} \times 5)$. The separated organic layer was combined and washed by brine $(20 \mathrm{~mL} \times 1)$, dried by anhydrous magnesium sulfate, and filtered. The filtrate was concentrated in vacuo, and the resulting residue was purified by silica gel column chromatography (EtOAc: $n$-hexane $=1: 10)$ to get pure thiiranes $(\mathbf{2 a - h})$. 
2-Benzyloxymethylthiirane $(\mathbf{2 a}){ }^{17}(0.29 \mathrm{~g}, 89 \%$, by irritating with $160 \mathrm{~W}, 6$ min) was obtained as colorless liquid, $\mathrm{R}_{\mathrm{f}}=0.69$ (EtOAc: $n$-hexane $\left.=1: 2\right),{ }^{1} \mathrm{H}-\mathrm{NMR}\left(\mathrm{CDCl}_{3}, 200 \mathrm{MHz}\right) \delta 2.18(\mathrm{dd}, J$ $\left.=5.2,1.2 \mathrm{~Hz}, 1 \mathrm{H}, \mathrm{H}_{\mathrm{a}}-3\right), 2.48\left(\mathrm{dd}, J=6.0,1.2 \mathrm{~Hz}, 1 \mathrm{H}, \mathrm{H}_{\mathrm{b}}-3\right), 3.08(\mathrm{~m}, 1 \mathrm{H}, \mathrm{H}-2), 3.47(\mathrm{dd}, J=$ 10.8, 6.4 Hz, 1H, Ha-4), 3.66 (dd, $J=10.8,6.0 \mathrm{~Hz}, 1 \mathrm{H}, \mathrm{Hb}-4), 4.56$ (s, 2H, OC$\left.\underline{H}_{2} \mathrm{C}_{6} \mathrm{H}_{5}\right), 7.33$ (m, $5 \mathrm{H}, \mathrm{ArH}) ;{ }^{13} \mathrm{C}-\mathrm{NMR}\left(\mathrm{CDCl}_{3}, 50 \mathrm{MHz}\right) \delta 23.7,32.1,73.0,74.5,127.6,127.7,128.3,137.8$; EIMS (70eV) m/z $181\left(\mathrm{M}^{+1}, 68\right), 163$ (30), 108 (40), 93 (42), 91 (100), 79 (17); HRMS (EI, m/z): Calcd for $\mathrm{C}_{10} \mathrm{H}_{12} \mathrm{OS}$ : 180.0603 . Found: 180.0603 .

2-Phenoxymethylthiirane $(\mathbf{2 b}){ }^{14 \mathrm{e}}(0.30 \mathrm{~g}, 90 \%$, by irritating with $130 \mathrm{~W}, 5 \mathrm{~min})$ was obtained as colorless liquid, $\mathrm{R}_{\mathrm{f}}=0.54$ (EtOAc: $n$-hexane $\left.=1: 6\right),{ }^{1} \mathrm{H}-\mathrm{NMR}\left(\mathrm{CDCl}_{3}, 200 \mathrm{MHz}\right) \delta 2.31$ (dd, $\left.J=5.2 \mathrm{~Hz}, 1.2 \mathrm{~Hz}, 1 \mathrm{H}, \mathrm{H}_{\mathrm{a}}-3\right), 2.59$ (dd, $\left.J=6.0,1.2 \mathrm{~Hz}, 1 \mathrm{H}, \mathrm{H}_{\mathrm{b}}-3\right), 3.26$ (m, 1H, H-2), 3.88 (dd, $J$ $\left.=10.2,6.8 \mathrm{~Hz}, 1 \mathrm{H}, \mathrm{H}_{\mathrm{a}}-4\right), 4.19\left(\mathrm{dd}, J=10.2 \mathrm{~Hz}, 5.2 \mathrm{~Hz}, 1 \mathrm{H}, \mathrm{H}_{\mathrm{b}}-4\right), 6.93(\mathrm{~m}, 3 \mathrm{H}, \mathrm{ArH}), 7.28(\mathrm{~m}$, $2 \mathrm{H}, \mathrm{ArH}) ;{ }^{13} \mathrm{C}-\mathrm{NMR}\left(\mathrm{CDCl}_{3}, 50 \mathrm{MHz}\right) \delta 23.8,31.3,72.3,114.4,121.0,129.4,158.2$; EI-MS (70eV) m/z $166\left(\mathrm{M}^{+}, 37\right), 133$ (17), 120 (10), 105 (7), 91 (100), 91 (4), 77 ( 6 ), 75 (20), 73 (100); HRMS (EI, $m / z)$ : Calcd for $\mathrm{C}_{9} \mathrm{H}_{10} \mathrm{OS}: 166.0447$, Found: 166.0449.

2-Phenylthiirane $(\mathbf{2 c}){ }^{14 \mathrm{e}}(0.30 \mathrm{~g}, 88 \%$, by irritating with $160 \mathrm{~W}, 5 \mathrm{~min})$ was obtained as colorless liquid, $\mathrm{R}_{\mathrm{f}}=0.83($ EtOAc: $n$-hexane $=1: 6),{ }^{1} \mathrm{H}-\mathrm{NMR}\left(\mathrm{CDCl}_{3}, 200 \mathrm{MHz}\right) \delta 2.65(\mathrm{dd}, J=$ $\left.5.8,1.4 \mathrm{~Hz}, 1 \mathrm{H}, 1 \mathrm{H}, \mathrm{H}_{\mathrm{a}}-3\right), 2.87\left(\mathrm{dd}, J=6.6 \mathrm{~Hz}, 1.4 \mathrm{~Hz}, 1 \mathrm{H}, \mathrm{H}_{\mathrm{b}}-3\right), 3.89(\mathrm{dd}, J=5.8,6.6 \mathrm{~Hz}, 1 \mathrm{H}$, $\mathrm{H}-2), 7.28(\mathrm{~m}, 5 \mathrm{H}, \mathrm{ArH}) ;{ }^{13} \mathrm{C}-\mathrm{NMR}\left(\mathrm{CDCl}_{3}, 50 \mathrm{MHz}\right) \delta 27.3,36.1,126.7,127.6,128.3,128.6$; EI-MS (70eV) m/z $136\left(\mathrm{M}^{+}, 81\right), 135$ (100), 104 (14), 92 (44), 79 (15); HRMS (EI, m/z): Calcd for $\mathrm{C}_{8} \mathrm{H}_{8} \mathrm{~S}: 136.0341$, Found: 136.0342 .

2-(Naphthalen-1-yloxymethyl)thiirane $(\mathbf{2 d}) .{ }^{14 \mathrm{e}}(0.29 \mathrm{~g}, 90 \%$, by irritating with $300 \mathrm{~W}, 45 \mathrm{~min})$ was obtained as colorless liquid, $\mathrm{R}_{\mathrm{f}}=0.55$ (EtOAc: $n$-hexane $\left.=1: 5\right),{ }^{1} \mathrm{H}-\mathrm{NMR}\left(\mathrm{CDCl}_{3}, 200\right.$ $\mathrm{MHz}) \delta 2.29\left(\mathrm{dd}, J=5.2,1.2 \mathrm{~Hz}, 1 \mathrm{H}, \mathrm{H}_{\mathrm{a}}-3\right), 2.54\left(\mathrm{dd}, J=6.2,1.2 \mathrm{~Hz}, 1 \mathrm{H}, \mathrm{H}_{\mathrm{b}}-3\right), 3.30(\mathrm{~m}, 1 \mathrm{H}$, $\mathrm{H}-2), 3.99$ (dd, $\left.J=10.2,6.8 \mathrm{~Hz}, 1 \mathrm{H}, \mathrm{H}_{\mathrm{a}}-4\right), 4.22$ (dd, $\left.J=10.2,5.6 \mathrm{~Hz}, 1 \mathrm{H}, \mathrm{H}_{\mathrm{b}}-4\right), 6.66$ (d, $J=7.6$ $\mathrm{Hz}, 1 \mathrm{H}, \mathrm{ArH}), 7.28$ (t, J=8.0 Hz, 1H, ArH), $7.43(\mathrm{~m}, 3 \mathrm{H}, \mathrm{ArH}), 7.75$ (dd, $J=6.2,3.2 \mathrm{~Hz}, 1 \mathrm{H}$, $\mathrm{ArH}), 8.29(\mathrm{dd}, J=6.2,3.6 \mathrm{~Hz}, 1 \mathrm{H}, \mathrm{ArH}) ;{ }^{13} \mathrm{C}-\mathrm{NMR}\left(\mathrm{CDCl}_{3}, 50 \mathrm{MHz}\right) \delta 23.7,31.4,72.5,104.9$, 120.6, 121.9, 125.2, 125.4, 125.6, 126.4, 127.3, 134.4, 154.0; EI-MS (70eV) m/z $216\left(\mathrm{M}^{+}, 30\right)$, 215 (17), 184 (11), 144 (9), 117 (32), 115 (49), 89 (13), 74 (100); HRMS (EI, m/z): Calcd for $\mathrm{C}_{13} \mathrm{H}_{12} \mathrm{OS}: 216.0603$, Found: 216.0605 .

2-(4-Methoxyphenoxymethyl)thiirane (2e). ${ }^{14 \mathrm{e}}(0.29 \mathrm{~g}, 90 \%$, by irritating with $170 \mathrm{~W}, 10 \mathrm{~min})$ was obtained as colorless crystals, $\mathrm{mp} 63^{\circ} \mathrm{C}($ EtOAC $+n$-hexane $), \mathrm{R}_{\mathrm{f}}=0.63$ (EtOAc: $n$-hexane $=$ 1: 2), ${ }^{1} \mathrm{H}-\mathrm{NMR}\left(\mathrm{CDCl}_{3}, 200 \mathrm{MHz}\right) \delta 2.30\left(\mathrm{dd}, J=5.2,1.2 \mathrm{~Hz}, 1 \mathrm{H}, \mathrm{H}_{\mathrm{a}}-3\right), 2.59(\mathrm{dd}, J=6.2,1.2$ $\left.\mathrm{Hz}, 1 \mathrm{H}, \mathrm{H}_{\mathrm{b}}-3\right), 3.24(\mathrm{~m}, 1 \mathrm{H}, \mathrm{H}-2), 3.76\left(\mathrm{~s}, 3 \mathrm{H}, \mathrm{ArOCH}_{3}\right), 3.85$ (dd, J = 10.4, 7.2 Hz, 1H, $\left.\mathrm{H}_{\mathrm{a}}-4\right)$, $4.16\left(\mathrm{dd}, J=10.4,5.2 \mathrm{~Hz}, 1 \mathrm{H}, \mathrm{H}_{\mathrm{b}}-4\right), 6.84(\mathrm{~m}, 4 \mathrm{H}, \mathrm{ArH}),{ }^{13} \mathrm{C}-\mathrm{NMR}\left(\mathrm{CDCl}_{3}, 50 \mathrm{MHz}\right) \delta 23.9$, 31.5, 55.7, 73.5, 114.7, 115.9, 152.5, 154.2; EI-MS (70eV) m/z $196\left(\mathrm{M}^{+}, 26\right), 124$ (11), 123 (33), 109 (9), 96 (28), 73 (100); HRMS (EI, $m / z$ ): Calcd for $\mathrm{C}_{10} \mathrm{H}_{12} \mathrm{O}_{2} \mathrm{~S}$ : 196.0553, Found: 196.0555; Anal Calcd for $\mathrm{C}_{10} \mathrm{H}_{12} \mathrm{O}_{2} \mathrm{~S}: \mathrm{C}, 61.20 ; \mathrm{H}, 6.16 ; \mathrm{S}, 16.34$; Found: C, 61.18; H, 6.23; S, 16.50 .

2-(3,4-Dimethoxyphenoxymethyl)thiirane (2f). (0.29 g, 89\%, by irritating with $180 \mathrm{~W}, 10 \mathrm{~min})$ was obtained as colorless crystals, $\mathrm{mp} 46^{\circ} \mathrm{C}\left(\right.$ EtOAC $+n$-hexane), $\mathrm{R}_{\mathrm{f}}=0.47$ (EtOAc: $n$-hexane $=$ 1: 6), ${ }^{1} \mathrm{H}-\mathrm{NMR}\left(\mathrm{CDCl}_{3}, 200 \mathrm{MHz}\right) \delta 2.32\left(\mathrm{dd}, J=5.2,1.4 \mathrm{~Hz}, 1 \mathrm{H}, \mathrm{H}_{\mathrm{a}}-3\right), 2.60(\mathrm{dd}, J=6.2,1.4$ 
$\left.\mathrm{Hz}, 1 \mathrm{H}, \mathrm{H}_{\mathrm{b}}-3\right), 3.25(\mathrm{~m}, 1 \mathrm{H}, \mathrm{H}-2), 3.83\left(\mathrm{~s}, 3 \mathrm{H}, \mathrm{ArOCH}_{3}\right), 3.85$ (s, 3H, $\left.\mathrm{ArOCH}_{3}\right), 3.87$ (dd, $J=$ $\left.10.2,5.6 \mathrm{~Hz}, 1 \mathrm{H}, \mathrm{H}_{\mathrm{a}}-4\right), 4.15\left(\mathrm{dd}, J=10.2,5.6 \mathrm{~Hz}, 1 \mathrm{H}, \mathrm{H}_{\mathrm{b}}-4\right), 6.39(\mathrm{dd}, J=8.8,2.8 \mathrm{~Hz}, 1 \mathrm{H}$, $\mathrm{ArH}), 6.55(\mathrm{~d}, J=2.8 \mathrm{~Hz}, 1 \mathrm{H}, \mathrm{ArH}), 6.76(\mathrm{~d}, J=8.8 \mathrm{~Hz}, 1 \mathrm{H}, \mathrm{ArH}) ;{ }^{13} \mathrm{C}-\mathrm{NMR}\left(\mathrm{CDCl}_{3}, 50 \mathrm{MHz}\right)$ $\delta 23.8,31.4,55.8,56.3,73.1,101.0,104.0,111.5,143.8,149.8,152.9$; EI-MS (70 eV), $226\left(\mathrm{M}^{+}\right.$, 83), 194 (17), 155 (53), 153 (41), 125 (55), 110 (16), 75 (38), 74 (100); HRMS (EI, m/z): calcd for $\mathrm{C}_{11} \mathrm{H}_{14} \mathrm{O}_{3} \mathrm{~S}$ : 226.0658. Found: 226.0659. Anal calcd for $\mathrm{C}_{11} \mathrm{H}_{14} \mathrm{O}_{3} \mathrm{~S}$ : C, 58.38; $\mathrm{H}, 6.24 ; \mathrm{S}$, 14.17. Found: C, 58.39; H, 6.26; S, 14.16 .

2-(3-Isopropoxy-4-methoxyphenoxymethyl)thiirane (2g). (0.26 g, 80\%), by irritating with 160 $\mathrm{W}, 10 \mathrm{~min}$ ) was obtained as colorless crystals, $\mathrm{mp} 37^{\circ} \mathrm{C}\left(\right.$ EtOAC $+n$-hexane), $\mathrm{R}_{\mathrm{f}}=0.58$ (EtOAc: $n$-hexane = 1: 3), ${ }^{1} \mathrm{H}-\mathrm{NMR}\left(\mathrm{CDCl}_{3}, 200 \mathrm{MHz}\right) \delta 1.37(\mathrm{~d}, J=6.0 \mathrm{~Hz}, 6 \mathrm{H}, \operatorname{ArOCHMe} 2), 2.31$ (dd, $J=5.6,1.2 \mathrm{~Hz}, 1 \mathrm{H}, \mathrm{Ha}-3), 2.59$ (dd, $J=6.2,1.2 \mathrm{~Hz}, 1 \mathrm{H}, \mathrm{Hb}-3), 3.25$ (m, 1H, H-2), 3.80 (s, 3H, $\left.\mathrm{ArOCH}_{3}\right), 3.85\left(\mathrm{dd}, J=10.2,7.0 \mathrm{~Hz}, 1 \mathrm{H}, \mathrm{H}_{\mathrm{a}}-4\right), 4.14\left(\mathrm{dd}, J=10.2,5.6 \mathrm{~Hz}, 1 \mathrm{H}, \mathrm{H}_{\mathrm{b}}-4\right), 4.51$ (hept, $J=6.0 \mathrm{~Hz}, 1 \mathrm{H}, \operatorname{ArOC} \underline{\mathrm{HMe}} 2), 6.40(\mathrm{dd}, J=8.8,3.0 \mathrm{~Hz}, 1 \mathrm{H}, \operatorname{ArH}), 6.56(\mathrm{~d}, J=3.0 \mathrm{~Hz}, 1 \mathrm{H}$, $\operatorname{ArH}), 6.78(\mathrm{~d}, J=8.8 \mathrm{~Hz}, 1 \mathrm{H}, \mathrm{ArH}) ;{ }^{13} \mathrm{C}-\mathrm{NMR}\left(\mathrm{CDCl}_{3}, 50 \mathrm{MHz}\right) \delta 22.0,23.9,28.8,31.4,56.6$, 71.3, 73.2, 104.8, 105.1, 112.8, 148.2, 152.8, 175.7; EI-MS (70eV) m/z $254\left(\mathrm{M}^{+}, 36\right), 222$ (15), 212 (27), 182 (22), 165 (39), 140 (47), 139 (63),111 (43), 73 (100); HRMS (EI, m/z): Calcd for $\mathrm{C}_{13} \mathrm{H}_{18} \mathrm{O}_{3} \mathrm{~S}$ : 254.0971, Found: 254.0973; Anal Calcd for $\mathrm{C}_{13} \mathrm{H}_{18} \mathrm{O}_{3} \mathrm{~S}: \mathrm{C}, 61.39$; H, 7.13; S, 12.61; Found: C, 61.51; H, 7.29; S, 12.69 .

4-(3,4-Dimethoxyphenylmethyl)thiirane (2h). (0.29 g, 90\%, by irritating with $200 \mathrm{~W}, 40 \mathrm{~min})$ was obtained as colorless liquid, $\mathrm{R}_{\mathrm{f}}=0.52$ (EtOAc: $n$-hexane $=1: 6$ ), ${ }^{1} \mathrm{H}-\mathrm{NMR}\left(\mathrm{CDCl}_{3}, 200\right.$ $\mathrm{MHz}) \delta 2.26\left(\mathrm{dd}, J=5.4,1.2 \mathrm{~Hz}, 1 \mathrm{H}, \mathrm{H}_{\mathrm{a}}-3\right), 2.54\left(\mathrm{dd}, J=6.2,1.2 \mathrm{~Hz}, 1 \mathrm{H}, \mathrm{H}_{\mathrm{b}}-3\right), 2.80(\mathrm{dd}, J=$ 14.1, 7.0 Hz, 1H, Ha-4), 2.91 (dd, $J=14.1,5.7$ Hz., 1H, Hb-4), 3.04 (m, 1H, H-2), 5.94 (s, 2H, $\left.\mathrm{OC}_{2} \mathrm{O}-\right), 6.73(\mathrm{~m}, 3 \mathrm{H}, \mathrm{ArH}) ;{ }^{13} \mathrm{C}-\mathrm{NMR}\left(\mathrm{CDCl}_{3}, 50 \mathrm{MHz}\right) \delta 25.6,36.0,42.2,100.9,108.2$, 109.0, 121.5, 133.0, 146.3, 147.6; EI-MS (70eV) m/z $194\left(\mathrm{M}^{+}, 100\right), 161$ (36), 135 (29), 131 (24), 103 (19); HRMS (EI, m/z): Calcd for $\mathrm{C}_{10} \mathrm{H}_{12} \mathrm{OS}$ : 194.0396. Found: 194.0399.

\section{Acknowledgements}

We are grateful to NSC, Taiwan for financial support, to Prof. C. C. Tseng Kaohsiung Medical University for lending microwave reactors, to Mrs. Chi-Jia Wang for taking NMR spectra, and to Mr. Min-Yuan Hung for running GC-EI/MS.

\section{References}

1. (a) Kudo, H.; Makino, S.; Kameyama, A.; Nishikubo, T. Macromolecules 2005, 38, 5964. (b)Kameyama, A.; Murakami, Y.; Nishikubo, T. Macromolecules 1999, 35, 1407. (c) Imai, T.; Hayakawa, K.; Satoh, T.; Kaga, H.; Kakuchi, T. Polym. Sci. Pol. Chem. 2002, 40, 3443.

2. (a) Gottarelli, G.; Mariani, P.; Spada, G. P.; Samori, B.; Forni, A.; Solladie, G.; Hibert, M. 
Tetrahedron, 1983, 39, 1337. (b) Scherowsky, G.; Gay, J. Liq. Cryst. 1989, 5, 1253.

3. (a) Chino, K.; Suga, K.; Ikawa, M.; Satoh, H. J. Appl. Polym. Sci. 2001, 82, 29537. (b). Kadoma Y. Dent. Mater. J. 2002, 21, 156.

4. (a) Goethals, E. J. Polym. Sci. Pol. Rev. 1968, 2, 73. (b) Fokin, A. V.; Allakhvediev, M. A.; Kolomiets, A. F.; Russ. Chem. Rev. (Engl. Transl.) 1990, 59, 705.

5. Testero, S. A.; Lee, M.; Staran, R. T.; Espahbodi, M.; Llarrull, L. I.; Toth, M.; Mobashery, S.; Chang, M. Med. Chem. Lett. 2011, 2, 177.

6. Sally, A. H.; Stephen, P. B.; Joel, L.; Peter J. S. Bioorg. Med. Chem. 2004, 12, 4877.

7. Cho, H. J.; Jung, M. J.; Kwon, Y.; Na, Y. Bioorg. Med. Chem. Lett. 2009, 19, 6766.

8. Kellis, J. T. Jr.; Childers, W. E.; Robinson, C. H.; Vickery, L. E. J. Biol. Chem. 1987, 262, 4421.

9. Lautenschlaeger, F. K.; Schwartz, N. V. J. Org. Chem. 1969, 34, 3991.

10. Woodgate, P. D.; Lee, H. H.; Rutledge, P. S.; Cambie, R. C. Tetrahedron Lett. 1976, 18, 1531.

11. Meyers, A. I.; Ford, M. E.; Tetrahedron Lett. 1975, 16, 2861.

12. Di Nunno, L.; Franchini, C.; Nacci, A.; Scilimati, A.; Sinicropi, M. S. Tetrahedron-Asymmetr. 1999, 10, 1913.

13. (a) Tamami, B.; Kolahdoozan, M. Tetrahedron Lett., 2004, 45, 1535; (b) Iranpoor, N.; Kazemi, F. Tetrahedron Lett., 1997, 53, 11377; (c) Iranpoor, N.; Kazemi, F. Synthesis 1996, 821; (d) Kazemi, F.; Kiasat, A. R. Phosphorus Sulfur 2003, 178, 1333; (e) Kazemi, F.; Kiasat, A. R.; Ebrahimi, S. J. Chem. Res. Synop. 2002, 4, 176; (f) Mohammadpoor-Baltork, Iraj; Khosropour, Ahmad R. Molecules, 2001, 6, 996; (g) Mirkhani, V.; Tangestaninejad, S.; Alipanah, L. Synth. Commun. 2002, 32, 621; (h) Iranpoor, N.; Adibi, H. Bull. Chem. Soc. Jpn. 2000, 73, 675; (i) Mohammadpoor-Baltork, I.; Khosropoor, A. R. Indian J. Chem. Sect. B. 1999, 38, 605; (j) Iranpoor, N.; Tamami, B.; Shekarriz, M. Synth. Commun. 1999, 29, 3313; (k) Mohammadpoor-Baltork, I.; Aliyan, H. Synth. Commun. 1998, 28, 3943; (1) Iranpoor, N.; Zeynizadeh, B. Synth. Commun. 1988, 28, 3913.

14. (a) Yadav, J. S.; Reddy, B. V. S.; Reddy, Ch. Srinivas; Rajasekhar, K. J. Org. Chem. 2003, 68, 2525; (b) Yadav, J. S.; Reddy, B. V. S.; Baishya, G. Syn. Lett. 2003, 3, 396; (c) Brimeyer, M. O.; Mehrota, A.; Quici, S.; Nigam, A.; Regen, S. L. J. Org. Chem. 1980, 45, 4254; (d) Blatt, A. H. Org. Synth. Coll.; Vol. IV; 1963; 232. (e) Reddy, C. S.; Nagavani, S. Heteroatom Chem. 2008, 19, 97.

15. (a) Kazemi, F.; Kiasat, A. R.; Ebrahimi, S. Synth. Commun. 2003, 33, 595. (b) Mohammadpoor-Baltork, I.; Aliyan, H.; J. Chem. Res. Synop. 2000, 3, 122; (c) Tangestaninejad, S.; Mirkhani, V. Synth. Commun. 1999, 29, 2079. (d) Kazami, F.; Kiasat, A. R.; Ebrahim, S. Phosphorus Sulfur 2001, 176, 135. (e) Zeynizadeh, B.; Baradarani, M. M.; Eisavi, R. Phosphorus Sulfur 2011, 186, 2208. (f) Eisavi, R.; Zeynizadeh, B.; Baradarani, M. M. Phosphorus Sulfur 2011, 186, 1902.

16. (a) Kaboudin, B.; Norouzi, H. Tetrahedron Lett. 2004, 45, 1283; (b) Iranpoor, N.; Firouzabadi, H.; Shekarize, M. Org. Biomol. Chem. 2003, 1, 724; (c) Takido, T.; Kobayashi, 
Y.; Itabashi, K. Synthesis, 1986, 9, 779; (d) Tangestaninejad, S.; Mirkhani, V. J. Chem. Res. Synop. 1999, 6, 370. (e) Wu, L.; Yang, L.; Fang, L.; Yang, C.; Yan, F. Phosphorus Sulfur 2010, 185, 2159. (f) Wu, Liqiang; Wang, Yongxue; Yan, Fulin; Yang, C. Bull. Korean Chem. Soc. 2010, 31, 1419.

17. Brugat, N.; Duran, J.; Polo, A.; Real, J.; lvarez-Larena, A.; Piniella, J. F. TetrahedronAsymmetr. 2002, 13, 569. 